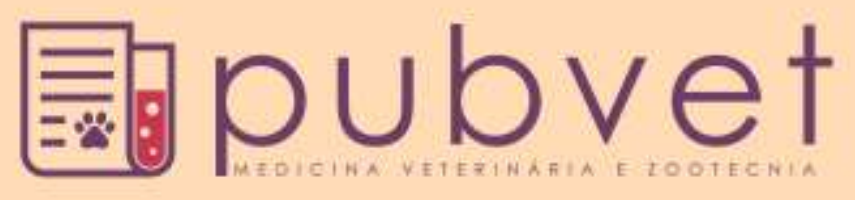

HTTP://DX.DOI.ORG/10.22256/PUBVET.V11N3.256-56

\title{
Principais patologias relacionadas aos efeitos adversos do uso de fármacos contraceptivos em gatas em Teresina - PI
}

\author{
Estéfane Kelly Dias Araújo ${ }^{1 *}$, Vanessa de Meneses Moura², Tiago Gonçalves Azevedo da \\ Fonseca Honório ${ }^{1}$, Rômulo Aécio Alves ${ }^{2}$, Ana Paula Barros Fonseca ${ }^{3}$, Marcelo Campos \\ Rodrigues $^{4}$, Roseli Pizzigatti Klein ${ }^{4}$
}

${ }^{1}$ Graduando (a) em Medicina Veterinária, Universidade Federal do Piauí (UFPI). Teresina, Piauí, Brasil.

${ }^{2}$ Médico (a) Veterinário (a), Universidade Federal do Piauí (UFPI). Teresina, Piauí, Brasil.

${ }^{3}$ Medica Veterinária, Residente em patologia clínica, Universidade Federal do Piauí (UFPI). Teresina, Piauí, Brasil.

${ }^{4}$ Docente do curso de Medicina Veterinária da Universidade Federal do Piauí-Teresina-PI - Brasil.

*Autor para correspondência, E-mail: estefane-kelly@hotmail.com

RESUMO. Cães e gatos são os principais animais de estimação na maioria das cidades. Quando não há interesse dos tutores na reprodução animal torna-se oportuno a apresentação pelo médico veterinário, dos métodos contraceptivos disponíveis, as vantagens e desvantagens de cada um. São eles por meios cirúrgicos ou por medicação hormonal, alvo de estudo desse trabalho. Os progestágenos são análogos sintéticos da progesterona e mimetizam os efeitos do corpo lúteo, e são utilizados na fêmea felina para a supressão ou adiamento do estro. Normalmente na gata a progesterona é um hormônio produzido pelo corpo lúteo e também pela placenta para manutenção da prenhez. O objetivo desse estudo foi analisar quantitativamente a relação da idade, uso de fármacos contraceptivos e as principais patologias associadas em gatas. As patologias foram: aborto, piometra, neoplasia mamária e hiperplasia mamária em gatas atendidas no período de janeiro de 2012 a julho de 2014 no Hospital Veterinário Universitário Jeremias Pereira da Silva, da Universidade Federal do Piauí.

Palavras chaves: Aborto, fármacos contraceptivos, hiperplasia mamária, neoplasia mamária, piometra

\section{Main pathologies related adverse effects of the use of contraceptive drugs in cats in Teresina - PI}

\begin{abstract}
Dogs and cats are the main pets in most cities. When there is no interest of the tutors in the animal reproduction it is opportune the presentation by the veterinary, the contraceptive methods available, the advantages and disadvantages of each one. They are by surgical means or by hormonal medication, the object of study of this work. Progestogens are synthetic analogs of progesterone and mimic the effects of the corpus luteum, and are used in feline females for the suppression or postponement of estrus. Normally in cats, progesterone is a hormone produced by the corpus luteum and by the placenta for maintenance of pregnancy. The objective of this study was to quantitatively analyze the relationship of age, contraceptive use and the main associated pathologies in cats. The pathologies were: abortion, pyometra, mammary neoplasia and mammary hyperplasia in cats treated from January 2012 to July 2014 at the University Veterinary Hospital Jeremias Pereira da Silva, Federal University of Piauí.
\end{abstract}

Keywords: Abortion, contraceptive drugs, mammary hyperplasia, mammary neoplasia, piómetra 


\section{Principales patologías relacionadas a los efectos adversos de la utilización de medicamentos anticonceptivos en gatas en Teresina - PI}

RESUMEN. Perros y gatos son los principales animales domésticos en la mayoría de las ciudades. Cuando no hay interés de los tutores en la reproducción animal se torna oportuna la presentación por el veterinario, de los métodos anticonceptivos disponibles, las ventajas y desventajas de cada uno. Son por medios quirúrgicos o medicamentos hormonales, objeto de estudio de este trabajo. Las progestágenos son análogos sintéticos de progesterona e imitan los efectos del cuerpo lúteo, y se utilizan en la hembra felina para la supresión o postergación del estro. Normalmente en la gata la progesterona es una hormona producida por el cuerpo lúteo y también por la placenta para mantenimiento de la preñez. El objetivo de este estudio fue cuantificar la relación de la edad, uso de fármacos anticonceptivos y las principales patologías asociadas en gatas. Las patologías fueron: aborto, piometra, cáncer de mama y la hiperplasia de mama en las gatas atendidas en el periodo de enero 2012 hasta julio 2014 en el Hospital de Veterinaria Universitario Jeremías Pereira da Silva, de la Universidad Federal de Piauí.

Palabras clave: aborto, fármacos anticoncepcionales, hiperplasia de mamaria, cáncer de mama, piometra

\section{Introdução}

Cães e gatos são, na maioria das cidades, os principais animais de estimação das famílias. Inerente a essa relação muitos temas tornam-se relevantes a fim de proporcionar bem-estar a ambos. Como por exemplo, o controle de natalidade dos animais, quando não houver interesse dos tutores em reproduzi-los.

Os métodos contraceptivos devem ser apresentados pelo médico veterinário aos proprietários, que devem ser alertados sobre vantagens e desvantagens de cada método, para que tomem a decisão entre contracepção cirúrgica ou a contracepção química (Reichmann, 2000).

A puberdade nas gatas geralmente ocorre em 910 meses de idade. São poliéstricas continuas em regiões próximas à Linha do Equador (Johnston et al., 1996). A atividade sexual é iniciada com o aumento do número de horas luz por dia e a inatividade sexual ocorre quando o número de horas decresce (Pope, 2000). A gata pode apresentar cio a cada 21 dias, na estação reprodutiva. A gestação dura aproximadamente dois meses (60 a 63 dias), com uma média de ninhada de aproximadamente quatro filhotes.

Normalmente na gata a progesterona é um hormônio produzido pelo corpo lúteo e também pela placenta para manutenção da prenhez. Os progestágenos são análogos sintéticos que mimetizam os efeitos do corpo lúteo, e são utilizados na fêmea para a supressão ou adiamento do estro. Os fármacos contraceptivos podem provocar efeitos secundários, como: inibição da imunidade uterina, proliferação das glândulas endometriais com formação de hiperplasia cística do endométrio, sendo efeitos contribuintes desenvolver piometra, hiperplasia mamária, diabetes mellitus, morte fetal e, possivelmente, neoplasia mamaria (Kustritz, 2011, Montanha et al., 2012) devendo serem utilizados com certa precaução, particularmente levando-se em consideração o ciclo estral. Por exemplo, é contra indicado o uso de fármacos contraceptivos para gatas nas fases proestro, estro e metaestro, pois, não impedem a prenhez e podem provocar a morte fetal pelo deficiente relaxamento da cérvix. (Montanha et al., 2012).

A perda fetal (aborto) ocorre na fase de desenvolvimento fetal, quando o feto não é independentemente viável. Na gata, a vida útil do corpo lúteo é longa no animal não gravídico e semelhante à gravidez. Quando a morte fetal ocorre, o corpo lúteo pode se manter e os produtos do concepto podem ficar retidos por aproximadamente o período normal de gestação. Portanto a autólise fetal é comum de ocorrer, podendo a mumificação e a maceração ser possíveis resultados da morte fetal (Zachary et al., 2012). Em uma gata gestante com descarga vulvar ou sinais sistêmicos de doença, deve-se fazer análise do histórico, exame físico completo, ultrasom abdominal, exame de sangue para medir a concentração de progesterona sérica e hemograma, e coleta de uma amostra de descarga 
vulvar para cultura aeróbica (Kustritz, 2011, Nelson and Couto, 2015).

A piometra é a infecção bacteriana do útero devido a alterações hormonais no período do diestro em gatas não castradas (Nunes et al., 2010). O comprometimento de outros órgãos não reprodutivos (principalmente rins e fígado) torna a piometra uma das maiores causas de atendimento ambulatorial, tratamento cirúrgico e óbito na casuística reprodutiva em pequenos animais. Por isso, deve ser considerada com demasiada atenção e o conhecimento de suas características, formas e manifestações clínicas conhecidas de todos os veterinários que trabalham com pequenos animais (Martins, 2007).

Neoplasia mamária é o terceiro tipo de neoplasia mais comum em gatas. Mais de $90 \%$ dos casos notificados são malignos, com propagação local e para os linfonodos regionais, pulmões e outros tecidos. Vários estudos ao longo de décadas têm demonstrado um efeito protetor da ovariosalpingohisterectomia ( $\mathrm{OSH})$, pois tem sido relatado que as gatas fêmeas sexualmente intactas, têm sete vezes o risco de desenvolver um tumor mamário quando comparado com gatas castradas (Birchard and Sherding, 2008, Kustritz, 2011). O uso indiscriminado de progestágenos para o controle do ciclo estral e as pseudogestações é cada vez mais responsável pelo aparecimento precoce de tumores mamários em cadelas e gatas (Sá, 2008).

A Hiperplasia mamária felina (hiperplasia fibroepitelial, fibroadenoma, fibroadenomatose) é uma doença mediada por progesterona, não neoplásica, normalmente encontrada em gatas jovens, após o estro (silencioso), em gestantes, com pseudogestação ou em animais que recebem progestágenos exógenos (Loretti et al., 2004, Nelson and Couto, 2015, Daleck et al., 2008).

O objetivo desse estudo foi analisar quantitativamente a relação de idade e as principais patologias associadas ao uso de fármacos contraceptivos em gatas. As patologias foram: aborto, piometra, neoplasia mamária e hiperplasia mamária.

\section{Material e Métodos}

O presente estudo foi desenvolvido no Hospital Veterinário Universitário (HVU) Jeremias Pereira da Silva, da Universidade Federal do Piauí (UFPI) com o objetivo de associar as principais patologias acometidas em gatas após o uso de fármacos contraceptivos.
Os dados obtidos se referem aos anos de 2012, 2013 e de janeiro a julho de 2014, totalizando dois anos e 7 meses.

Foram avaliadas as fêmeas felinas, com idade variando entre 5 meses a 15 anos, observando-se três raças: siamês, persa e animais sem raça defina (SRD). De acordo com seus prontuários de atendimento clínico, foi constando os seguintes diagnósticos definitivos: aborto, piometra, neoplasia mamária e hiperplasia mamária. As informações referentes à anamnese (idade, raça, sinais clínicos, uso de contraceptivos), exames hematológicos, bioquímicos, citológicos, histopatológico, métodos de diagnóstico por imagem, terapia e prognóstico também foram considerados.

\section{Resultados e Discussão}

Durante 2012, 2013 e janeiro a julho de 2014 foram diagnosticados 80 casos de piometra (25\%), 39 casos de neoplasia mamária (12\%), 153 casos de abortos (49\%) e 44 casos de hiperplasia mamária (14\%).

Pode-se observar que a quantidade de gatas que apresentou aborto, teve destaque, pois a porcentagem equivale praticamente à metade de todos os casos analisados. Em segundo lugar estão os diagnósticos para piometra, em terceiro e quarto lugar tem-se neoplasia mamária e hiperplasia mamária respectivamente.

Dos 153 casos de abortos, 56\% usavam contraceptivos e somente $4 \%$ não fizeram uso (Gráfico 1).

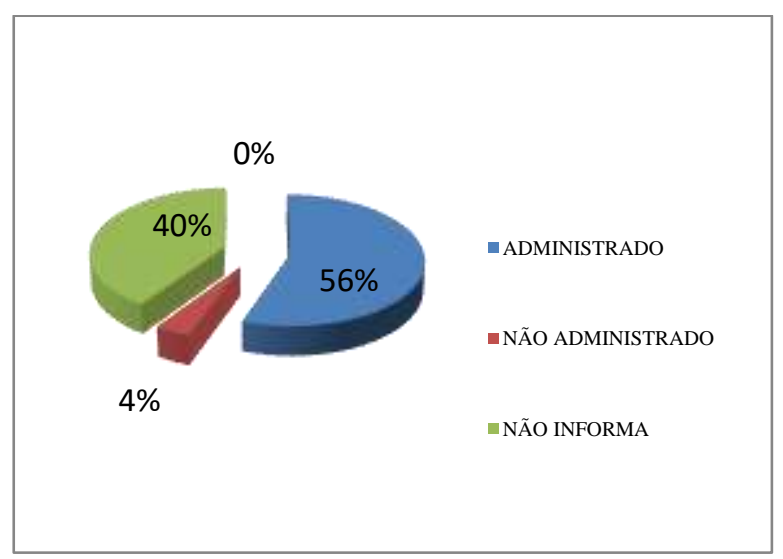

Gráfico 1. Frequência do uso de fármacos contraceptivos nos casos de diagnóstico de aborto em gatas, de janeiro de 2012 a julho de 2014, no HVU - UFPI.

De acordo com Nelson and Couto (2015), o aborto é um evento isolado sem causa identificável, onde, $4 \%$ de casos que ocorreram 
sem o uso de anticoncepcional, observados no presente relato corroboram com tal informação. $\mathrm{O}$ uso indiscriminado de contraceptivos, feito sem orientação médica veterinária pode provocar a ocorrência de prenhez com risco de morte fetal pelo deficiente relaxamento da cérvix (Montanha et al., 2012), isso explica o alto percentual (56\%) encontrado no presente relato.

Dos 80 casos de piometra encontrados, 41 usavam fármacos contraceptivos (51\%), 6 não usavam (8\%) e 33 não havia informação se usavam ou não (41\%) (Gráfico 2).

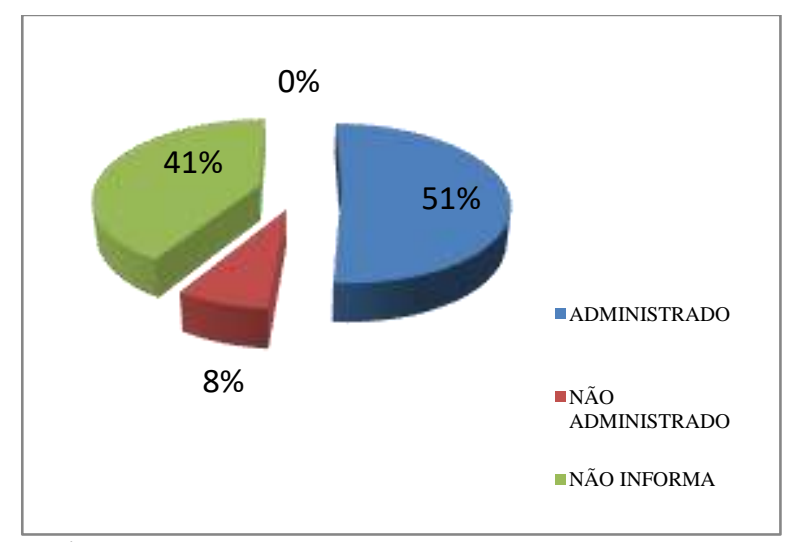

Gráfico 2. Frequência do uso de fármacos contraceptivos nos casos de diagnóstico de piometra em gatas, de janeiro de 2012 a julho de 2014, no HVU - UFPI

Mais da metade dos casos de piometra houve o relato dos tutores de fazer uso de fármacos contraceptivos. Esses dados estão de acordo com alguns estudos que afirmam que o uso de progestágenos exógenos para o controle do estro é considerado fator etiológico para a piometra (Dias et al., 2013, King and Boag, 2007).

Em estudo de histofometria realizado com gatas submetidas à OSH foi revelado resultados numéricos que confirmam que o uso de fármacos contraceptivos afetam a parede uterina em sua totalidade, contribuindo com desenvolvimento da doença (Kustritz, 2011).

A ocorrência de piometra de acordo com a idade foi de $21,25 \%$ dos casos com menos de 1 ano de idade, $46,25 \%$ de 1 ano aos 5 anos, $20 \%$ de 6 a 10 anos e 3,75\% de 11 a 15 anos. Onde a faixa etária com maior número de casos foi entre 1 a 5 anos. Esses dados condizem com os de Kustritz (2011) o qual observou que a piometra é comum em gatas mais velhas e que há uma probabilidade significativa que elas venham a apresentar a doença uterina por volta de 5 anos de idade. Além disso, esses resultados também se aproximam dos de Vines (2011) que observou em seu estudo que
$78 \%$ das fêmeas afetadas possuíam idade superior a 6 anos.

Essa pesquisa corrobora com os estudos de Evangelista et al. (2011) os quais concluíram que animais jovens que fazem uso de anticoncepcional, se tornam mais susceptíveis a piometra, embora seja mais comum em animais adultos e idosos.

Dos 39 casos de neoplasia mamária, 23 usavam fármacos contraceptivos (59\%), 3 não usavam (8\%) e 13 não havia informação (33\%) (Gráfico 3). Essas informações estão de acordo com os observados por Daleck et al. (2008) onde afirmam haver alta relação entre o uso prévio de fármacos que contém progestágeno sintético ou combinações de estrógeno e progestágeno e o desenvolvimento de neoplasias mamárias benignas ou malignas. E também com Dias et al. (2013) que afirmam que os hormônios possuem papel importante no desenvolvimento de neoplasia mamária.

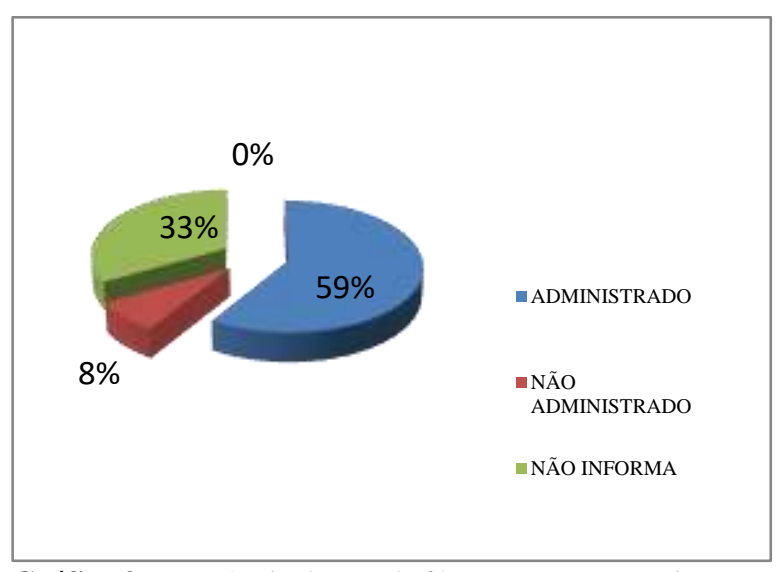

Gráfico 3. Frequência do uso de fármacos contraceptivos nos casos de diagnóstico de neoplasia mamária em gatas, de janeiro de 2012 a julho de 2014, no HVU - UFPI.

Conforme a idade, os casos de neoplasia mamária foram observados em $35,9 \%$ dos animais com menos de 1 ano de idade, $15,38 \%$ entre 1 ano e 5 anos, $33,33 \%$ entre 6 e 10 anos e $15,38 \%$ de 11 a 15 anos. Quanto ao uso de contraceptivos, o surgimento de neoplasias mamárias foi mais evidente na faixa etária entre 6 e 10 anos, sendo que, o surgimento de tal afecção dentre as que não usaram anticonceptivos ou não informaram seu uso, a maioria dos casos acometeu em gatas mais jovens. Essas neoplasias são mais comuns em gatas idosas, com média de idade de 10 a 12 anos (Mialot, 1984, Daleck et al., 2008, Birchard and Sherding, 2008, Nelson and Couto, 2015) e a menor incidência desta patologia está entre os $8 \mathrm{e}$ 11 anos (́́, 2008) ou entre 8 e 10 anos (Queiroga and Lopes, 2002). 
Dos 44 casos de hiperplasia mamária, 57\% usavam fármacos contraceptivos, $10 \%$ não os usavam e $33 \%$ não havia informação sobre o uso de progestágenos (Gráfico 4). Esses dados refletem os dados de alguns estudos que demonstram que a hiperplasia mamária normalmente acontece em gatas que recebem progestágenos exógenos. (Loretti et al., 2004, Daleck et al., 2008, Filgueira et al., 2008, Nelson and Couto, 2015).

Para hiperplasia mamária foram encontrados de acordo com a idade 59,09\% dos casos em gatas com menos de 1 ano de idade, $31,81 \%$ de 1 ano aos 5 anos, 2,28\% de 6 a 10 anos e $6,81 \%$ dos casos de 11 a 15 anos. A faixa etária que apresentou maior incidência foi até 1 ano de idade. A Hiperplasia mamária felina é uma doença normalmente encontrada em gatas jovens (Daleck et al., 2008, Nelson and Couto, 2015), dados semelhantes aos encontrados nesse estudo.

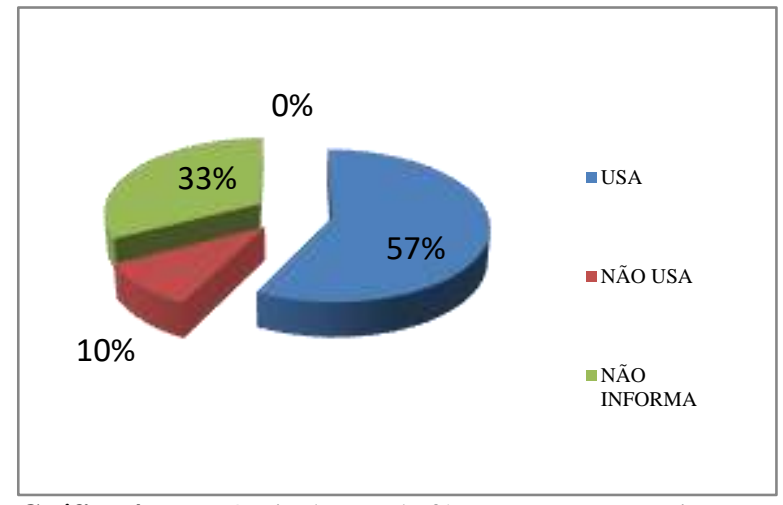

Gráfico 4. Frequência do uso de fármacos contraceptivos nos casos de diagnóstico de hiperplasia mamária em gatas, de janeiro de 2012 a julho de 2014, no HVU - UFPI.

Esse estudo foi realizado a partir de dados presentes no histórico clínico dos pacientes. De acordo com Malacarne et al. (2008), a ficha clínica, provavelmente, seja o documento mais importante e muitas vezes o único, para comprovar o estado inicial do paciente, onde deve incluir a identificação completa do paciente, procedimentos cirúrgicos, queixa principal relatada pelo proprietário do paciente, o histórico de saúde do animal e ainda, deve conter os sinais clínicos, entre outros dados relevantes.

\section{Conclusão}

Esse estudo apresentou resultados referentes ao uso de fármacos anticoncepcionais, patologias associadas ao uso, relacionando também a idade. Conclui-se que o uso indiscriminado de fármacos contraceptivos é frequente, bem como, é grande a quantidade de animais que apresentam efeitos adversos decorrente do seu uso. Isso reafirma e contribui com outros trabalhos já realizados. É importante que a população, principalmente àqueles que possuem animais de estimação sejam conscientizados dos perigos que o uso desses produtos traz para a saúde e o bem-estar animal. Para isso, é fundamental que os médicos veterinários informem aos proprietários que a esterilização cirúrgica constitui a principal medida de controle mais segura contra patologias e proles indesejadas.

\section{Referências Bibliográficas}

Birchard, S. J. \& Sherding, R. G. 2008. Manual Saunders: clínica de pequenos animais, São Paulo.

Daleck, C. R., De Nardi, A. B., Rodigheri, S. M. \& Motta, F. R. 2008. Neoplasia do sistema urinário. Roca, São Paulo.

Dias, L. G. G. G., Oliveira, M. E., Dias, F. G. G., Calazans, S. G. \& Confort, V. A. 2013. Uso de fármacos contraceptivos e seu efeitos adversos em pequenos animais. Enciclopedia Biosfera, 9, 2077.

Evangelista, L. S. M., Quessada, A. M., Lopes, R. R. F. B., Alves, R. P. A., Gonçalves, L. M. F. \& Drumond, K. O. 2011. Perfil clínico e laboratorial de gatas com piometra antes e após ovário-histerectomia. Revista Brasileira de Reprodução Animal, 35, 347-351.

Filgueira, K. D., Costa, R., Paulo Fernando Cisneiros \& Paula, V. V. 2008. Hiperplasia mamária felina: sucesso terapêutico com o uso do aglepristone. Ciência Animal Brasileira, 9, 1010-1016.

Johnston, S. D., Root, M. V. \& Olson, P. N. S. 1996. Ovarian and testicular function in the domestic cat: clinical management of spontaneous reproductive disease. Animal Reproduction Science, 42, 261-274.

King, L. G. \& Boag, A. 2007. BSAVA manual of canine and feline emergency and critical care. British small animal veterinary association.

Kustritz, M. V. R. 2011. Clinical canine and feline reproduction: evidence-based answers. John Wiley \& Sons.

Loretti, A. P., Ilha, M. R. S., Breitsameter, I. \& Faraco, C. S. 2004. Clinical and pathological study of feline mammary fibroadenomatous change associated with depot medroxyprogesterone acetate therapy. Arquivo 
Brasileiro de Medicina Veterinária e Zootecnia, 56, 270-274.

Malacarne, P., Langer, M., Nascimben, E., Moro, M. L., Giudici, D., Lampati, L., Bertolini, G. \& Medicine, I. G. f. t. E. o. I. i. I. C. 2008. Building a continuous multicenter infection surveillance system in the intensive care unit: findings from the initial data set of 9,493 patients from 71 Italian intensive care units. Critical Care Medicine, 36, 1105-1113.

Martins, D. G. 2007. Complexo hiperplasia endometrial cística/piometra em cadelas: fisiopatogenia, características clínicas e laboratoriais e abordagem terapêutica. Animal. Universidade estadual Paulista, Jaboticabal.

Mialot, J. P. 1984. Patologia da reprodução dos carnívoros domésticos. A Hora veterinária, Porto Alegre.

Montanha, F. P., Corrêa, C. S. d. S. \& Parra, T. C. 2012. Maceração fetal em gata em decorrência do uso de contraceptivos - Relato de caso. Revista Científca Eletrônica de Medicina Veterinária, 10, 1-6.

Nelson, R. W. \& Couto, C. G. 2015. Medicina interna de pequenos animais. Elsivier Editora, Amsterdan.

Nunes, J. K. P., Scorsato, P. S. \& Repetti, C. S. F. 2010. Hormonioterapia em tumores mamários de cadelas. UNIMAR, 19, 31-39.

Pope, C. E. 2000. Embryo technology in conservation efforts for endangered felids. Theriogenology, 53, 163-174.
Queiroga, F. \& Lopes, C. 2002. Tumores mamários caninos, pesquisa de novos factores de prognóstico. Revista Portuguesa de Ciências Veterinárias, 97, 119-127.

Reichmann, M. d. L. A. B. 2000. Controle de populaçôes de animais de estimação. In: Técnico, M. (ed.). Instituto Pasteur, São Paulo.

Sá, F. N. 2008. Citologia e histopatologia: a sua importância no diagnóstico de tumores mamários em canídeos e felídeos. Faculdade de Medicina Veterinária. Universidade Técnica de Lisboa, Lisboa.

Vines, G. M. G. 2011. Estudo do complexo hiperplasia quística do endométrio-piómetra na gata doméstica. Faculdade de Medicina Veterinária. Universidade Técnica de Lisboa, Lisboa.

Zachary, J. F., McGavin, D. \& McGavin, M. D. 2012. Bases da patologia em veterinária. Elsevier Brasil, Rio de Janeiro.

\section{Article History:}

Received 8 December 2016

Accepted 16 January 2016

Available on line 14 February 2017

License information: This is an open-access article distributed under the terms of the Creative Commons Attribution License 4.0, which permits unrestricted use, distribution, and reproduction in any medium, provided the original work is properly cited. 